UCRL-JC-121681

PREPRINT

\title{
Preliminary Uranium Enrichment Analysis Results Using Cadmium Zinc Telluride Detectors
}

\author{
A. D. Lavietes \\ J. H. McQuaid \\ T. J. Paulus
}

This paper was prepared for submittal to the 9th International Workshop on Room Temperature Semiconductor $X$ - and Gamma-Ray Detectors, Associated Electronics and Applications Grenoble, France

September 18-22, 1995

September 8, 1995

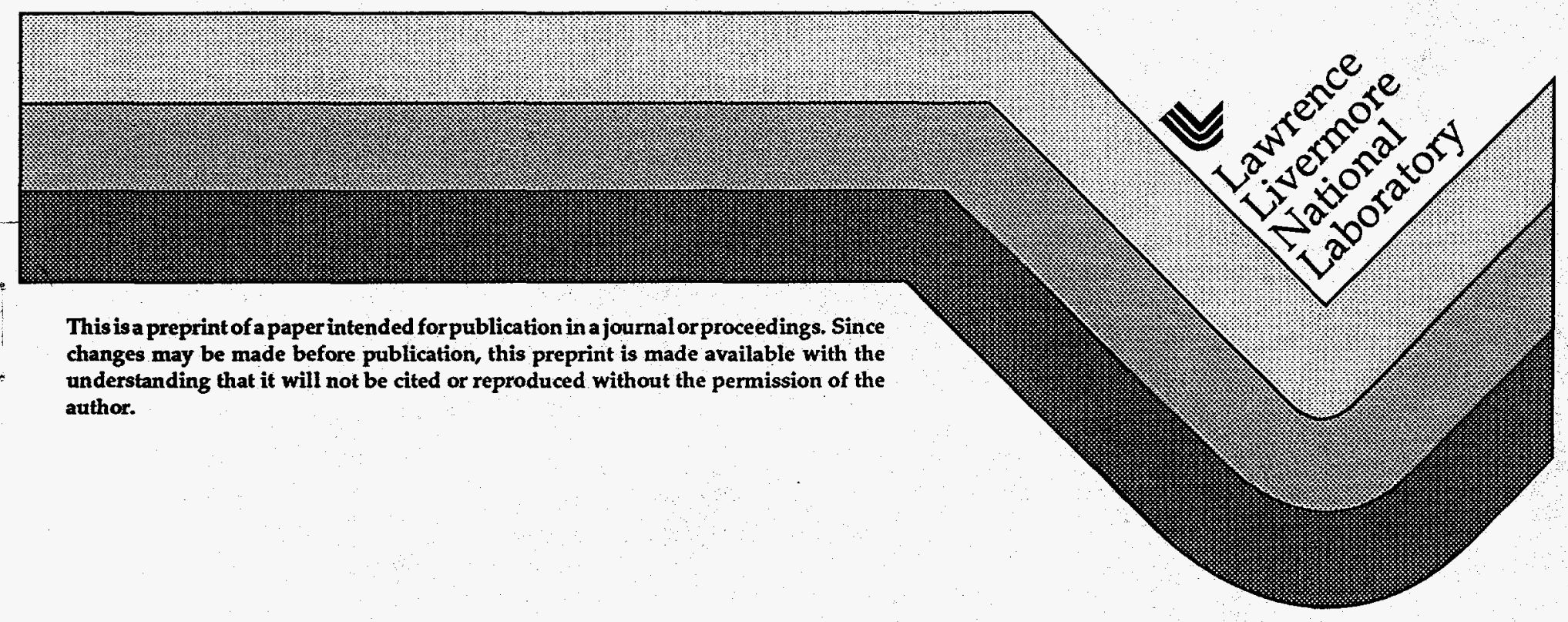

\section{MASTER}




\section{DISCLAIMER}

This document was prepared as an account of work sponsored by an agency of the United States Government. Neither the United States Government nor the University of California nor any of their employees, makes any warranty, express or implied, or assumes any legal liability or responsibility for the accuracy, completeness, or usefuiness of any information, apparatus, product, or process disclosed, or represents that its use would not infringe privately owned rights. Reference herein to any specific commercial product, process, or service by trade name, trademark, manufacturer, or otherwise, does not necessarily constitute or imply its endorsement, recommendation, or favoring by the United States Government or the University of California. The views and opinions of authors expressed herein do not necessarily state or reflect those of the United States Government or the University of California, and shall not be used for advertising or product endorsement purposes.

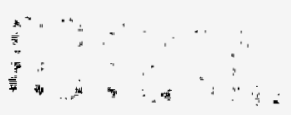




\section{DISCLAIMER}

Portions of this document may be illegible in electronic image products. Images are produced from the best available original document. 


\title{
Preliminary Uranium Enrichment Analysis Results Using Cadmium Zinc
}

\section{Telluride Detectors ${ }^{1}$}

\author{
Anthony D. Lavietes
}

Lawrence Livermore National Laboratory, 7000 East Ave. L-230, Livermore, CA 94550 USA

James H. McQuaid

Lawrence Livermore National Laboratory, 7000 East Ave. L-230, Livermore, CA 94550 USA

T.J. Paulus

EG\&G ORTEC, 100 Midland Road, Oak Ridge, TN 37831 USA

\begin{abstract}
Lawrence Livermore National Laboratory (LLNL) and EG\&G ORTEC have jointly developed a portable ambient-temperature detection system that can be used in a number of application scenarios. The detection system uses a planar cadmium zinc telluride (CZT) detector with custom-designed detector support electronics developed at LLNL and is based on the recently released MicroNOMAD multichannel analyzer (MCA) produced by ORTEC. Spectral analysis is performed using software developed at LLNL that was originally designed for use with high-purity germanium (HPGe) detector systems. In one application, the CZT detection system determines uranium enrichments ranging from less than $3 \%$ to over $75 \%$ to within accuracies of $20 \%$. The analysis was performed using sample sizes of $200 \mathrm{~g}$ or larger and acquisition times of $30 \mathrm{~min}$. We have demonstrated the capabilities of this system by analyzing the spectra gathered by the CZT detection system from uranium sources of several enrichments. These experiments demonstrate that current CZT detectors can, in some cases, approach performance criteria that were previously the exclusive domain of larger HPGe detector systems.
\end{abstract}

\section{Introduction}

Reliable high-precision radionuclide analyses have historically been performed using LN cooled high-purity germanium (HPGe) detector systems. The $\mathrm{x}$ - and gamma-ray spectra from these systems are then analyzed by one of several computer routines developed to determine the radionuclide content of a source. Typically, many energy lines are grouped close together (less than $1 \mathrm{keV}$ apart), which dictates the use of high-resolution radiometric instrumentation such as HPGe detectors in order to resolve these individual energies.

To perform spectral measurements and analysis using lower-resolution detection systems, analysis software packages must be intelligent enough to resolve the complex structure of closely spaced energy lines. This includes the software characterization of low-energy tailing discussed later. These software programs typically will self-calibrate to known peak locations and employ other user information to perform a curve or peak fitting routine to a specified energy range in a given spectrum. The curve fit will iteratively conform to known relative intensities and other statistical information until a reasonably good fit is achieved. An example of the results of this process for an HPGe detsctor spectrum of uranium is shown in Figure 1.

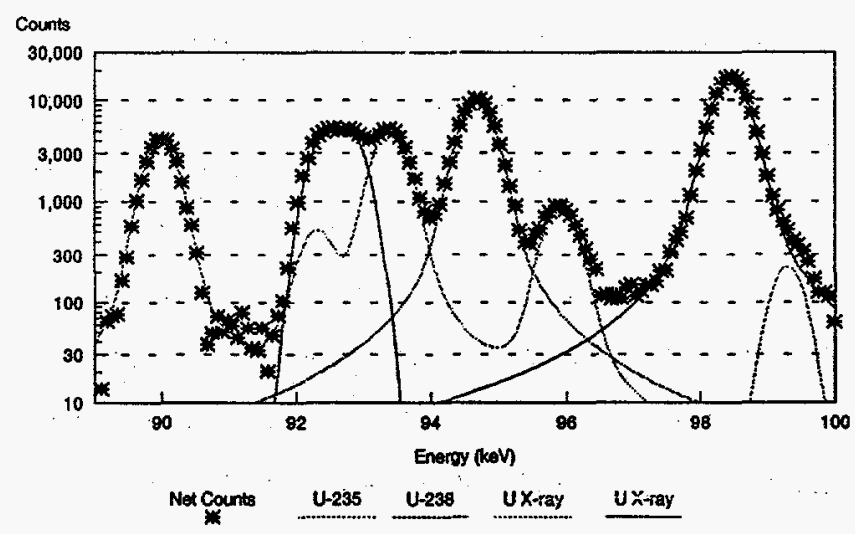

Figure 1. GRPANL Analysis Results of a Uranium Spectrum from a High Resolution LEPS Detector ( $484 \mathrm{eV}$ at $100 \mathrm{keV})$.

\footnotetext{
${ }^{1}$ This work performed under the auspices of the U.S. Department of Energy by the Lawrence Livermore National Laboratory under Contract W-7805-Eng-48. LLNL Document UCRL-JC-121681.
} 
Several widely used -software packages that perform uranium enrichment analyses for HPGe detector spectra have been developed at LLNL. These packages are based on the GRPANL software package developed by Ray Gunnink and Wayne Ruhter [1]. Of recent interest is the current effort to tailor this software to the spectral characteristics of moderateresolution ambient-temperature detectors such as cadmium telluride (CdTe) and CZT. The resulting spectrum from these detectors is somewhat less resolved than a typical HPGe detector spectrum, yet significantly better than the widely used sodium iodide (NaI) detector. A relative qualitative comparison of the spectra from each detector type is shown in Figure 2.

The focus of this work is to readily demonstrate the suitability of CZT detectors for uranium enrichment measurements in both laboratory and field applicaticns. While CZT detector spectra approach the resolution of HPGe detectors, the resolution is limited by a severe low-energy tailing phenomenon that is characteristic of high $\mathrm{Z}$ ambienttemperature semiconductor detectors and, even more importantly, the efficiency of a large ( $5 \times 5 \times 5-\mathrm{mm}) \mathrm{CZT}$ detector is much lower with respect to a HPGe detector due to this smaller relative size. In an effort to perform a more accurate analysis of CZT detector spectra, LLNL is working to develop a better model for the low-energy tailing phenomenon. This will ideally allow the analysis software to perform a better peak fit and result in more accurate enrichment data.

The benefits of having a portable instrument are significantly reduced if it requires an unusually long period of time to gather and analyze the required data. It would seem obvious that to reduce this problem the largest volume CZT detector should be used. The problem with this approach is that the performance of these detectors drops off rather sharply as the volume is increased. This is primarily due to the characteristically large amount of charge trapping and transport problems intrinsic to the material (hole transport is particularly poor, contributing heavily to the low-energy tailing problem). Due to these trapping and transport issues, it does not take much detector thickness to exceed the mean free path of the charge carriers and significantly affect the charge collection. Increasing the detector bias voltage helps

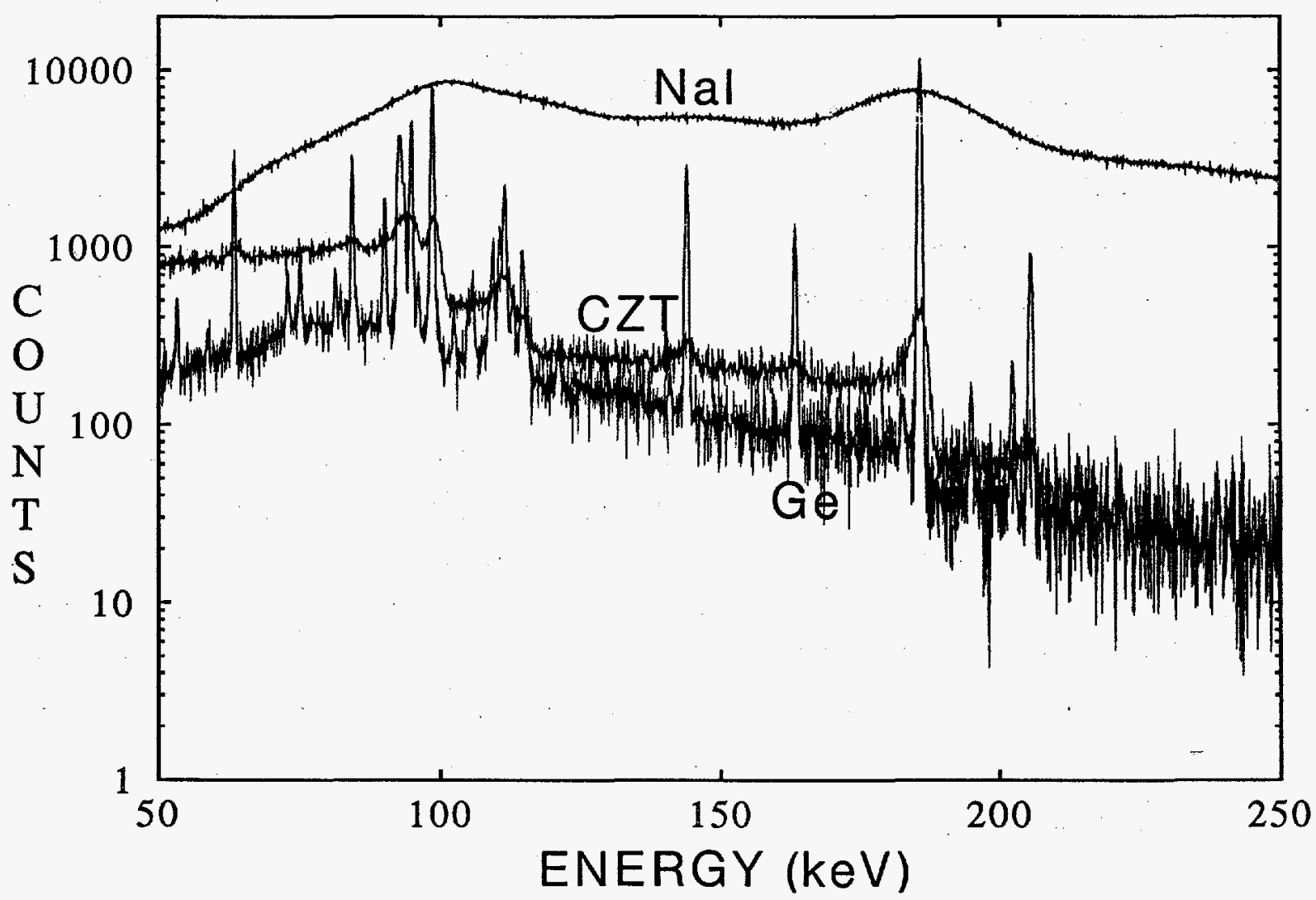

Figure 2. Comparison of Acquired Spectra - HPGe, NaI and CZT Detectors. 
improve charge collection somewhat, but is limited by the detector resistivity and corresponding leakage current and resulting shot noise.

Earlier work [2] was performed in an effort to determine the necessary detector performance that allows the use of unmodified HPGe detector spectral analysis software (GRPANL) to operate reliably on CZT detector spectra. This performance specification was determined to be roughly $3 \%$ full width at half maximum (FWHM) resolution at $122 \mathrm{keV}$ $\left({ }^{57} \mathrm{Co}\right)$. The detector geometry can then be determined based on the performance specification, allowing for a variety of implementations. A better low-energy tailing model that more accurately portrays a CZT detector spectrum will likely relax the performance specification, allowing for larger detector volumes and resulting higher efficiencies and smaller data acquisition times.

The experiments that were conducted to determine the resolution specification used 1-g uranium sources of known enrichments. While these were sufficient to analyze performance requirements, the experiments were quite lengthy and did not model a realistic situation. Due to selfabsorption of $x$ - and gamma-rays, larger sources do not exhibit a significant increase in emission. Therefore, a uranium source of $200 \mathrm{~g}$ or larger is required to accurately portray a realistic situation. Sources with very high or very low enrichments will still take more time, but the increased activity due to the larger sample size more than adequately compensates for this problem.

\section{Experiments}

The detector used for these experiments was a $5 \times 5 \times 5$ $\mathrm{mm}$ CZT detector (10\% molecular zinc) purchased from $\mathrm{eV}$ Products, Inc. This is a particularly high-performance detector giving a resolution of about 2.2\% FWHM at $122 \mathrm{keV}$ $\left({ }^{57} \mathrm{Co}\right)$. The detector was couplei to an ORTEC 142PC preamp modified to optinize the periormance of $\mathrm{CZT}$ detectors. The output of the 142PC was connected to an ORTEC 672 Spectroscopy Amplifier set for $0.5-\mu \mathrm{sec}$ shaping time. The output of the 672 was then connected to the ORTEC Micróî́TOMAD portabie MíCÁ.
The MicroNOMAD is a 1.5 -lb battery-powered portable gamma-ray spectrometer developed especially for this application. The unit consists of an amplifier suitable for NaI, $2 \mathrm{k}$ ADC, spectrum stabilizer, microprocessor and memories, as well as parallel, RS-232, and RS-485 ports. An internal amplifier is readily bypassed when using the CZT detector, which includes an optimized amplifier developed at LLNL. The MicroNOMAD unit is small, only $7 \times 7 \times 21-\mathrm{cm}$, and requires $<1.5 \mathrm{~W}$. It operates from $8 \mathrm{AA}$ batteries for over 8 hours. It can communicate with any desktop or portable PC with a parallel printer port using included software. The MicroNOMAD can be programmed and acquired spectra can be downloaded with very little effort.

Since the focus of this work was on portable instrumentation, a portable computer was used that also contained the MGA analytical software. MGA will operate from any PC platform running DOS with the speed of analysis directly related to the processor used in the system. A 486-based PC runs the analysis routine in only a few seconds.

Several uranium sources with a variety of enrichments were analyzed and are listed in Table 1 . It should be noted that all of the sources are $200 \mathrm{~g}$ or larger. It should also be noted that the minimum acquisition time necessary to obtain enough statistical information for a reliable analysis was not known. To determine this information while minimizing the total experiment time, many consecutive short-duration spectra were taken for each source. For higher-intensity sources, a series of consecutive 5-min spectra immediately followed by a series of 30-min spectra were obtained. The 5min spectra were omitted for the lower-intensity National

Table 1. GRPANL Analysis Results of Several Uranium Spectra Using a $5 \times 5 \times 5$-mm CZT Detector.

\begin{tabular}{|c|c|c|c|c|c|c|c|c|}
\hline 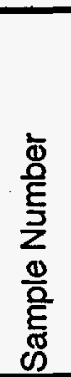 & 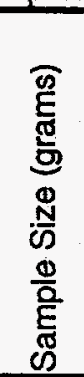 & 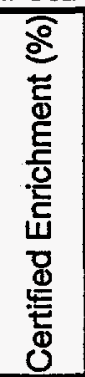 & 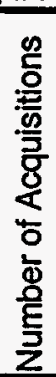 & 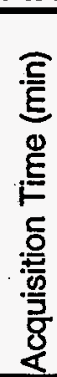 & $\frac{\widehat{a}}{3}$ & 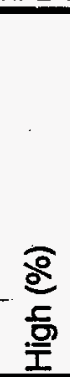 & 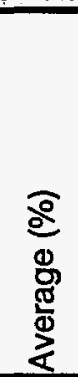 & 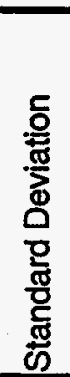 \\
\hline A2 & 2731 & 93.2 & $\overline{12}$ & 30 & 56.6 & 90.0 & 79.7 & 13.9 \\
\hline $\mathrm{C} 2$ & 1017 & 20 & 10 & 30 & 18.2 & 36.3 & 23.6 & 5.5 \\
\hline N1 & 200 & 2.95 & 16 & 30 & 2.0 & 5.3 & 3.6 & 0.8 \\
\hline N2 & 200 & 4.5 & $\overline{16}$ & 30 & 3.4 & 6.2 & 4.9 & 0.8 \\
\hline
\end{tabular}


Institute of Standards and Technology (NIST) standard sources. This process allowed for the summing of spectra until the statistics necessary for reliable analysis were achieved.

These experiments were conducted in two phases. The first phase consisted of gathering spectra for the two NIST uranium standards in a laboratory. Since these sources were at the 200-g limit, the activity was relatively small and required the detector to be placed in contact with the sample container. The system dead time was less than $5 \%$ for both sources. Several initial spectra were taken to verify that the system was operating properly prior to obtaining the series of 30-min spectra.

The second phase of the experiment was to gather the spectra in the same manner for the remaining sources at a remote location. The source-detector geometry for these acquisitions was set such that the system dead time was between 5 and 10\%. Typical 5- and 30-min spectra are shown in Figures 3 and 4, respectively.

The spectra were then analyzed using the GRPANL software. Before analyzing a spectrum, software variables were set to tailor the sensitivity of the software analysis routines to the low-energy tailing characteristics of CZT detectors. This process is purely empirical and is iterated until a satisfactory result is obtained. In this case reliable and repeatable close matches to the NIST standard sources were used to determine when the proper values had been selected. All of the spectra were then analyzed and the results are shown in Table 1.

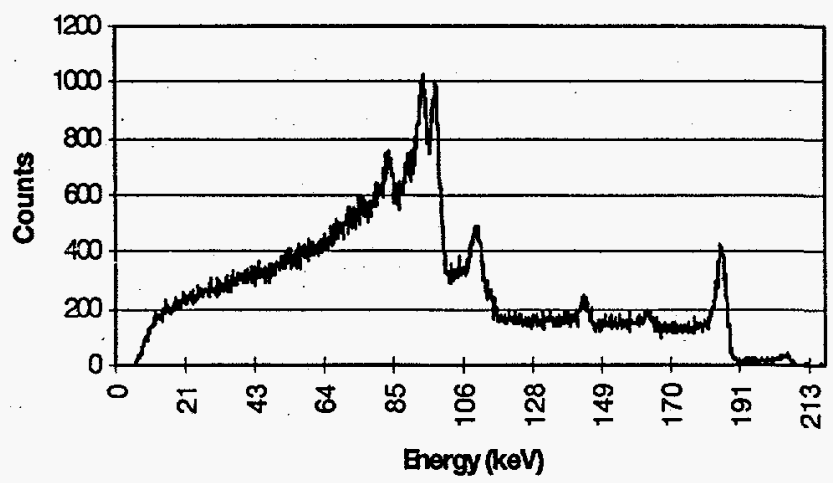

Figure 3. Uranium Spectrum - 5-Min Acquisition Time Using a 5 × 5 × 5-mm CZT Detector.

\section{Results}

All of the hardware used in these experiments performed flawlessly. The detector and associated electronics continuously provided stable spectra regardless of the length of acquisition. The equipment could be assembled in a matter of minutes and by using Job Control files (part of the MicroNOMAD operating software), entire experiment sequences could be performed unattended. As it was our intention to demonstrate the use of a completely portable instrument package, the LLNL-developed portable CZT detector unit became available at the end of the experiment and was used to duplicate portions of the initial work. The portable unit replaced the ORTEC 142PC preamp, 672 amplifier, the detector bias supply, and associated NIM bin and performed with virtually identical results. When using the LLNL CZT detector assembly, the portable computer (with GRPANL software) and MicroNOMAD were all that were required for a complete analysis system:

When the analysis work began, about half of the 5-min spectra and all but a few of the 30-min spectra individually gave results to within $20 \%$ of the advertised enrichment. This was surprising in that it was anticipated that several spectra would need to be summed to obtain enough statistical data to perform the analysis. The results were better with the longer acquisitions, but for this application as a first-order screening tool, these analysis results were very encouraging.

To determine uranium enrichment, the GRPANL software analysis routine ratios the ${ }^{235} \mathrm{U}$ gamma-ray peak at $93.36 \mathrm{keV}$

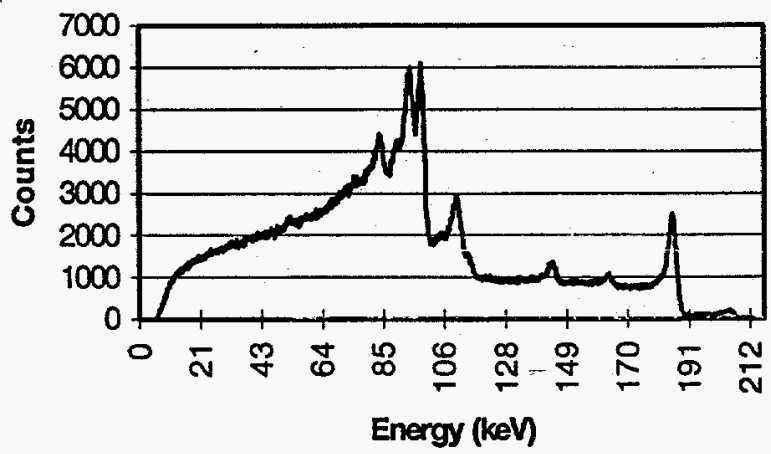

Figure 4. Uranium Spectrum - 30-Min Acquisition Time Using a $5 \times 5 \times 5-\mathrm{mm}$ CZT Detector. 
to the ${ }^{238} \mathrm{U}$ gamma-ray peak at $92.792 \mathrm{keV}$. For very high or very low enrichments, the low-abundance isotope results in an energy peak that cannot be resolved from background. This problem is exaggerated in spectra taken by moderate or poor-resolution detection systems. The result of using this analysis method is that the ability to determine the enrichment of uranium sources is best below about $90 \%$ or above about $2 \%$. This effect can be seen in the data presented in Table 1 for sample A2. This sample was taken from a source characterized at over $93 \%$ enrichment using a HPGe detector system.

Another problem occurred while analyzing the spectra involving analysis consistency. Two virtually identical spectra taken sequentially using identical sources and detection systems yielded vastly different results. This was best noted while analyzing the 30 -min spectra. It appeared that, at random, the analysis results would be exceedingly inaccurate. While the software did have less than desired overall performance, the random poor results distorted the statistical data (presented in Table 1), making the analysis software performance appear even worse.

\section{Conclusions}

The ability to determine uranium enrichments using only a 5-min data acquisition truly exceeded expectations. While the analysis software did not successfully analyze all of the short-duration data-acquisition spectra to within $20 \%$ of the actual enrichment, the results strongly indicate that with continued analytical software development, CZT detector systems can be used to perform this task adequately as a reliable first-order evaluation tool. This low-cost portable ambient-temperature detection instrument can be used in place of the standard LN-cooled HPGe detection system for applications requiring moderate resolution. This opens the door for many potential applications using this technology.

Tremendous increases in CZT detector performance have been noted in the past 2 years and it is now reasonable to expect resolution better than $3 \%$ FWHM at $122 \mathrm{keV}\left({ }^{57} \mathrm{Co}\right.$ ) from a $5 \times 5 \times 5$-mm CZT detector. The realization of this performance relies on the use of high-quality electronics and careful analysis methods. The FWHM reported in every case described in this paper was determined by the absolute method [3] to provide complete repeatability.

Because of moderate resolution and availability of larger volumes, CZT detectors have generated a significant interest for use in applications requiring reasonable efficiency. At the current state of the technology it is apparent that CZT detector systems cannot compete with the performance of HPGe detector systems in terms of resolution or efficiency, although they can be used as an effective first-order screening tool. Their low power requirements, light weight, and small size make these detectors likely candidates for moderateresolution portable instrumentation. In comparison, $\mathrm{NaI}$ has tremendous efficiency, but does have some temperature dependencies and, as can be seen in Figure 2, has a resolution that does not readily lend itself to spectral analysis.

While the software variables were being manipulated to optimize the operation with CZT detectors, it was noted that the spectra did not always give the same results. Since each of the spectra was obtained sequentially from the same source with identical detector-source geometries, this was somewhat puzzling. The software and the process of variable manipulation are still under development and the results we have obtained in this work should help to identify problems for further improvements.

\section{References}

[1] R. Gunnink, W.D. Ruhter. and J.B. Niday, "GRPANL: A Suite of Computer Programs for Analyzing Complex Ge and AlphaParticle Spectra," Lawrence Livermore National Laboratory, Livermore, CA, UCRL-53861, Vol. 1, May 1988.

[2] A.D. Lavietes, J.H. McQuaid, W.D. Ruhter, and T.J. Paulus, "Development of a Portable Ambient Temperature Radiometric Assaying Instrument," IEEE Trans. on Nucl. Sci. 42, No. 4, p. 634, August 1995.

[3] A.D. Lavietes and J.H. McQuaid, "Characterization Inconsistencies in CdTe and CdZnTe Gamma-Ray Detectors," Lawrence Livermore National Laboratory, Livermore, CA, UCRL-JC-117142, October 1994. 